\title{
Two Results on the Asymptotic Performance of Quantizers
}

\author{
JAMES A. BUCKLEW, MEMBER, IEEE
}

\begin{abstract}
A necessary and sufficient condition is presented for the normalized asymptotic $r$ th power distortion of a mismatched multidimensional quantizer to converge to a certain optimum constant known as Bennett's integral. The dimensionality of our results is allowed to approach infinity in order to make some universal source coding comparisons to quantization theory.
\end{abstract}

\section{INTRODUCTION}

A BLOCK quantizer is a function $Q_{N}(x)$ which maps $x \in \mathbb{R}^{k}$ into one of $N$ "output levels" $y_{1}, y_{2}, \cdots, y_{N}$ each in $\mathbb{R}^{k}$. The information rate at the output of a quantizer will be defined to be $(1 / k) \log N$. Suppose $X$ is a $k$-dimensional random vector. Let $E\{\cdot\}$ be the expectation operator. The performance of a quantizer in this paper will be measured by the $r$ th power distortion measure

$$
D=\frac{1}{k} E\left\{\left\|X-Q_{N}(X)\right\|^{r}\right\},
$$

where $\|\cdot\|$ denotes the usual $l_{2}$ norm and $r>0$. For a fixed $N$, the objective of an optimal quantizer design is to make the distortion as small as possible. Several authors [1]-[10] have considered various aspects of this problem and related questions dealing with optimum quantizcrs in some detail. When this best quantizer is implemented, the quantizer is said to be "matched" to the data.

In practice, however, we may not always be able to use a quantizer matched to the incoming data. Situations arise where signal statistics might have changed, incorrect assumptions about data statistics have occurred, or several data sources with different statistics might need to be digitized by a single quantizer. Hence it is of practical and theoretical interest to be able to predict the performance of a quantizer when it is not matched to the incoming data.

Perhaps the most ubiquitous reference dealing with this problem is W. R. Bennett's 1948 paper [11] which modeled a one-dimensional quantizer as a zero memory monotonic increasing nonlinearity $C(\cdot)$ (called the compressor) followed by a uniform $N$ level quantizer in turn followed by the inverse nonlinearity $C^{1}(\cdot)$ (called the expandor). The entire operation is called companding and any one-dimensional nonuniform quantizer can be implemented in this fashion. Bennett argued that the mean square distortion

Manuscript received August 10,1981; revised March 4, 1983. This work was presented at the 1982 IEEE International Information Theory Conference, Les Arcs, France, June 21-25, 1982.

The author is with the Electrical and Computer Engineering Department, University of Wisconsin, Madison, WI 53706. $(r=2)$ introduced by such a companding operation could be approximated by the expression

$$
D \cong \frac{\left(L_{2}-L_{1}\right)^{2}}{12 N^{2}} \int_{L_{1}}^{L_{2}} \frac{p(x)}{(\lambda(x))^{2}} d x,
$$

where $\lambda(x) \triangleq C^{\prime}(x), p(x)$ is the probability density function of the data with support contained in the uniform quantizer range interval $\left[L_{1}, L_{2}\right]$, and $N$ is assumed to be very large. Bennett's integral is a useful tool for predicting the asymptotic (large $N$ ) performance of a mismatched quantizer. Smith [4] extended Bennett's work by finding the optimal nonlincarity $C(\cdot)$ to minimize his distortion expression. His results were

$$
C(x)_{\mathrm{opt}}=\frac{\int_{-\infty}^{x} p(\alpha)^{1 / 3} d \alpha}{\int_{-\infty}^{\infty} p(\alpha)^{1 / 3} d \alpha}
$$

with resulting distortion of

$$
D \cong \frac{1}{12 N^{2}}\left(\int_{L_{1}}^{L_{2}} p(\alpha)^{1 / 3} d \alpha\right)^{3} .
$$

This result fitted in nicely with the work of Panter and Dite [1] who had derived the above distortion cxpression by means other than a companding approach. Algazi [5] essentially reproduced Bennett's and Smith's work for the more general $r$ th power distortion measure. Gersho [8] generalized Bennetl's expression to several dimensions and to $r$ th power distortion measures. Gersho's motivation for this generalization was to minimize Bennett's integral in order to obtain distortion expressions for optimal quantizers. No author to date has explicitly considered necessary and sufficient conditions for Bennett's integral to give the correct distortion.

Section II of this paper is devoted to notation and some preliminary results taken from other sources. Section III derives a necessary and sufficient condition for Bennett's integral to hold in $k$-dimensional space with $r$ th power distortion measures. We give a simple example in Appendix $B$ where Bennett's integral does not give the correct asymptotic distortion of a quantizer.

Many authors [18], [19], [20] have considered the problem of source coding a stationary (but not necessarily ergodic) information source. These researchers make use of a result due to Rohlin [17] which states (under certain conditions) that a stationary random process can be thought 
of as a mixture of ergodic random processes. From this "decomposition" result most of the nonergodic source coding theorems then follow by clever applications of the usual ergodic ones.

One of the more startling results to come from this "nonergodic" theory is the concept of universal source codes. One starts with the idea of an information source which consists of a mixture of ergodic subsources. Obviously, for every one of these ergodic subsources, there exists a source code that will get arbitrarily close to the optimal rate-distortion bound for that subsource. The basic result of universal source coding is that there exists a "univcrsal" source code that, when used to code a particular subsource, will get arbitrarily close to the rate-distortion bound for that source. In other words, one code works near optimally for all. For a deeper discussion of these concepts, the reader is referred to [20].

In Section IV we investigate the distortion performance of multidimensional mismatched quantizers as we let the dimensionality approach infinity. As a by-product of this we are then able to make a quantization theory analog to universal source coding.

\section{Notation and Preliminaries}

In the sequel $r$ will be a fixed positive number, $k$ will be a fixed positive integer, $\|\cdot\|$ will denote the $l_{2}$ norm on $\mathbb{R}^{k}$, $m^{k}$ will denote Lebesgue measure on $\mathbb{R}^{k}$. Let $b$ be a positive number. For any measurable function $h: \mathbb{R}^{k} \rightarrow$ $[0, \infty)$, let $\|h\|_{b}$ denote the integral of $h^{b}$ raised to the $1 / b$ power. For $F_{a c}$ and $F_{s}$ denote, respectively, the absolutely continuous and the singular components (with respect to $m^{k}$ ) of a distribution $F$. The derivative of $F_{a c}$ will be denoted by $f$. Let $\mathscr{Q}_{N}$ denote the class of all $N$ output level $k$-dimensional quantizers. Define

$$
B_{r}(N)=\inf _{Q \in \mathscr{Q}_{N}} N^{r / k} \int\|x-Q(x)\|^{r} d F(x)
$$

$\varlimsup$ im and lim will denote the limit superior and limit inferior, respectively.

We now state for reference a theorem to be used in the next two sections. The following theorem is proved in [3]. It is a generalization of a result by Zador [12], [13]. Note that any distribution function may be written $F=a F_{a c}+$ $(1-a) F_{s}$, where $a \in[0,1]$.

Theorem 1: If $E\left\{\|x\|^{r+\epsilon}\right\}<\infty$ for some $\epsilon>0$, then

$$
\lim _{N \rightarrow \infty} B_{r}(N)=a J_{k, r}\|f\|_{k / k+r},
$$

where $J_{k, r}$ is a universal constant depending only on $k$ and $r$.

Remarks: We will use this theorem in the succeeding sections only for absolutely continuous distributions. Hence from now on we may consider $a$ to be equal to one.

Gersho [8] and Zador [12], [13] present upper and lower bounds to $J_{k, r}$ for various values of $k$ and $r$. For example $J_{1,2}=1 / 12$ and $J_{2,2}=5 / 26 \sqrt{3}$.

\section{Mismatch Quantizer Performance}

Let $f(x)$ and $g(x)$ be two probability density functions on $\mathbb{R}^{k}$. We assume there exists a $\epsilon>0$ such that $\int_{\mathbb{R}^{k}}\|x\|^{r+\epsilon}(f(x)+g(x)) d x<\infty$. Let $\left\{Q_{N}(x)\right\}$ be a sequence of $N$ output level quantizers such that

$$
\lim _{N \rightarrow \infty} N^{r / k} \int_{\mathbb{R}^{k}}\left\|x-Q_{N}(x)\right\|^{r} g(x) d x=J_{k, r}\|g\|_{k / k+r},
$$

where we know such a sequence exists from Theorem 1. Now let us define

$$
\begin{aligned}
& M_{g}^{N}(E) \triangleq N^{r / k} \int_{E}\left\|x-Q_{N}(x)\right\|^{r} g(x) d x \\
& M_{g}(E) \triangleq J_{k, r}\left(\int_{E} g(x)^{k / k+r} d x\right)\left(\int_{\mathbb{R}^{k}} g^{k / k+r}(x) d x\right)^{r / k} .
\end{aligned}
$$

Obviously $M_{g}^{N}, M_{g}$ are measures on the Lebesgue measurable sets of $\mathbb{R}^{k}$. Our main result, Theorem 2 , follows almost immediately from Lemma 2 . The proof of Lemma 2 is a standard analysis argument and will be consigned to Appendix A.

Lemma 2:

$$
\lim _{N \rightarrow \infty} M_{g}^{N}(E)=M_{g}(E)
$$

for all Lebesgue measurable sets $E$ in $\mathbb{R}^{k}$.

We now state our main result.

Theorem 2: Suppose $\int_{\mathbb{R}^{k}}(f / g) d M_{g}<\infty$. Then the following limit

$$
\lim _{N \rightarrow \infty} N^{r / k} \int_{\mathbb{R}^{k}} f(x)\left\|x-Q_{N}(x)\right\|^{r} d x
$$

exists and equals

$$
J_{k, r}\left(\int_{\mathbb{R}^{k}} \frac{f(x)}{g(x)^{r / k+r}} d x\right)\left(\int_{\mathbb{R}^{k}} g(x)^{k / k+r} d x\right)^{r / k}
$$

if and only if

$$
\int_{\mathbb{R}^{k}} \frac{f}{g} d M_{g}^{N}
$$

is uniformly integrable over $N$, i.e.,

$$
\lim _{\alpha \rightarrow \infty} \sup _{N} \int_{\left\{x: \frac{f(x)}{g(x)} \geq \alpha\right\}} \frac{f}{g} d M_{g}^{N}=0 .
$$

Proof: Define $X_{N}$ to be the $k$-dimensional random variable with probability measure defined by $P\left(X_{N} \in B\right)$ $=M_{g}^{N}(B) / M_{g}^{N}\left(\mathbb{R}^{k}\right) ; X_{\infty}$ is the random variable with probability measure $P\left(X_{\infty} \in B\right)=M_{g}(B) / M_{g}\left(\mathbb{R}^{k}\right)$ for all Borel sets $B$. Now for any Borel set $A$,

$$
\begin{aligned}
P\left(\frac{f\left(X_{N}\right)}{g\left(X_{N}\right)} \in A\right) & =P\left(X_{N} \in\left(\frac{f}{g}\right)^{-1}(A)\right) \\
& =M_{g}^{N}\left(\left(\frac{f}{g}\right)^{-1}(A)\right) / M_{g}^{N}\left(\mathbb{R}^{k}\right) .
\end{aligned}
$$


Now take limit to find

$$
\begin{aligned}
\lim _{N \rightarrow \infty} P\left(\frac{f\left(X_{N}\right)}{g\left(X_{N}\right)} \in A\right) & =M_{g}\left(\left(\frac{f}{g}\right)^{-1}(A)\right) / M_{g}\left(\mathbb{R}^{k}\right) \\
& =P\left(\frac{f\left(X_{\infty}\right)}{g\left(X_{\infty}\right)} \in A\right) .
\end{aligned}
$$

Hence

$$
\frac{f\left(X_{N}\right)}{g\left(X_{N}\right)} \stackrel{D}{\rightarrow} \frac{f\left(X_{\infty}\right)}{g\left(X_{\infty}\right)}
$$

Hence from Theorem 5.4 in [14], we have that uniform integrability is necessary and sufficient for

$$
\begin{aligned}
E\left\{\frac{f\left(X_{N}\right)}{g\left(X_{N}\right)}\right\} & =\int_{\mathbb{R}^{k}} \frac{f}{g} d M_{g}^{N} \\
& =N^{r / k} \int_{\mathbb{R}^{k}} f(x)\left\|x-Q_{N}(x)\right\|^{r} d x \\
& \rightarrow \underset{N \rightarrow \infty}{\rightarrow} E\left\{\frac{f\left(X_{\infty}\right)}{g\left(X_{\infty}\right)}\right\}=\int_{\mathbb{R}^{k}} \frac{f}{g} d M_{g} \\
& =J_{k, r}\left(\int_{\mathbb{R}^{k}} \frac{f(x)}{g(x)^{r / k+r}} d x\right)\left(\int_{\mathbb{R}^{k}} g(x)^{k / k+r}\right)^{r / k} .
\end{aligned}
$$

We now have an obvious corollary.

Corollary: If $f(x) / g(x)$ is bounded, then

$$
\lim _{N \rightarrow \infty} \int_{\mathbb{R}^{k}} \frac{f}{g} d M_{g}^{N}=\int_{\mathbb{R}^{k}} \frac{f}{g} d M_{g} .
$$

Remarks: Consider the ratio

$$
\boldsymbol{R}_{E}=\int_{E} g(x)^{k / k+r} d x / \int_{\mathbb{R}^{k}} g(x)^{k / k+r} d x .
$$

In Appendix $A$ it is shown that this is the fraction of the total number of output levels of $Q_{N}(x)$ to be found contained in the set $E$. Let $E$ be an open hypercube. If we divide $\boldsymbol{R}_{E}$ by the Borel measure of $E$ and let $E$ collapse downward about some interior point $x$, we would obtain $[15$, p. 217]

$$
\begin{aligned}
\lim _{m^{k}(E) \rightarrow 0} \frac{\boldsymbol{R}_{E}}{m^{k}(E)} & =g(x)^{k, k+r} / \int_{\mathbb{R}^{k}} g(x)^{k / k+r} d x \\
& \triangleq \lambda(x), \quad m^{k} \text { almost everywhere, }
\end{aligned}
$$

a point density function for the quantizer output levels. Rewriting Theorem 2 with this point density function we obtain

$$
\lim _{N \rightarrow \infty} N^{r / k} \int_{\mathbb{R}^{k}} f(x)\left\|x-Q_{N}(x)\right\|^{r} d x=J_{k, r} \int_{\mathbb{R}^{k}} \frac{f(x)}{\lambda(x)^{r / k}} d x
$$

When $k=1$ this is the most familiar form of Bennett's integral. The general multidimensional integral above was first derived by Gersho [8]. Since the right-hand side of the above equation must equal the limit given in Theorem 2 we will also call the limit in Theorem 2 Bennett's integral. We do this even though the form of the integral appears quite different. We hope no confusion will follow from this convention.

In Appendix $\mathrm{B}$ we give an example of a sequence of quantizers $Q_{N}(x)$ that obtains the optimal coefficient for a one-dimensional uniform distribution on the interval $[0,1]$, i.e., $g(x)=1$. However, $\overline{\lim }_{N} \int N^{2}\left|x-Q_{N}(x)\right|^{2} f(x) d x=$ $\infty$ even though Bennett's integral is finite.

The generalized Fatou's lemma [16] implies that

$$
\frac{\lim }{N} \int_{\mathbb{R}^{k}} \frac{f}{g} d M_{g}^{N} \geq \int_{\mathbb{R}^{k}} \frac{f}{g} d M_{g} .
$$

Hence the limit on the right-hand side in the theorem statement is the best that we may achieve.

\section{INFINITE BLOCK LENGTH}

The preceeding results have all been based upon a fixed block length $k$ (the dimension of the quantizer). We now would like to compare some of our results with known performance bounds from rate distortion theory by letting $k$ approach infinite and taking $r=2$. Let $p_{k}(x)$ denote the $k$-dimensional probability density of a source. Define

$$
\bar{H} \triangleq-\varlimsup_{k} \frac{1}{k} \int p_{k}(x) \log p_{k}(x) d x
$$

as the differential entropy of the source. Zador [12] conjectured that $\left\|p_{k}\right\|_{k / k+2} \rightarrow e^{2 \bar{H}}$ as $k \rightarrow \infty$.

In [8], Gersho presents the proof to the following theorem which he attributes to Thomas Liggett.

Theorem 3: $\lim _{k \rightarrow \infty}\left\|p_{k}\right\|_{k / k+r}=\exp [r \bar{H}]$ if $p_{k}$ is the joint probability density of $x=\left(x_{1}, \cdots, x_{k}\right)$ where $\left\{x_{i}\right\}$ is a stationary ergodic process, $\bar{H}$ is the entropy of the process, and $\left\|p_{1}\right\|_{1 / 1+r_{0}}<\infty$ where $0<r<r_{0}$.

If $\int_{\mathbb{R}^{k}}\|x\|^{r+\delta} p(x) d x<\infty$ for some $\delta>0$ then $\|p\|_{k / k+r}$ $<\infty$ by Hölder's inequality. Hence in the above theorem we may replace the integral existence condition by a moment condition.

In the same paper Gersho removes the ergodic assumption and shows that $\lim _{k \rightarrow \infty}\left\|p_{k}\right\|_{k / k+2} \geq e^{2 \bar{H}}$. We would now like to further generalize these results. In [17] it is shown that any discrete time stationary source having a separable $\sigma$-algebra can be decomposed into a mixture of ergodic subsources. We then can have the following characterization for any such stationary measure on $\mathbb{R}^{\infty}$ :

$$
u(E)=\int_{\Lambda} u_{\alpha}(E) d W(\alpha),
$$

for some probability measure $W$ defined on suitable subsets of $\Lambda$ (a subset of the real line) and all Borel measurable $E$ in $\mathbb{R}^{\infty}$, where $\left\{u_{\alpha}\right\}_{\alpha \in \Lambda}$ is a collection of stationary ergodic sources with entropy $H_{\alpha}$. We remark that this is the same set up as in [19]; the reader is referred there for more details. Further assume that $\left\{u_{\alpha}\right\}_{\alpha \in \Lambda}$ restrictions to $k$ dimensional Euclidean space $\left\{u_{\alpha}^{k}\right\}_{\alpha \in \Lambda}$ satisfy for some $\epsilon>0$

$$
\sup _{\alpha \in \Lambda} \int_{\mathbb{R}^{k}}\|x\|^{r+\epsilon} d u_{\alpha}^{k}<M_{k} .
$$

Also suppose $u_{\alpha}^{k} \ll m^{k}$ for all $\alpha$ and denotc

$$
\frac{d u_{\alpha}^{k}}{d m^{k}} \triangleq f_{\alpha}(x)
$$

We assume $u^{k} \alpha$ is $\mathbb{R}^{k} \times W$ measurable for all $k$. Obviously

$$
u^{k}(E)=\int_{\Lambda} u_{\alpha}^{k}(E) d W(\alpha)
$$


for Borel measurable $E$ in $\mathbb{R}^{k}$. This implies that $u^{k} \ll m^{k}$. Define

$$
g(x) \triangleq \frac{d u^{k}}{d m^{k}}=\int_{\Lambda} f_{\alpha}(x) d W(\alpha) .
$$

Theorem 4: With the above assumptions and notation

$$
\lim _{k \rightarrow \infty}\|g\|_{k / k+r}=\int_{\Lambda} e^{r H_{\alpha}} d W(\alpha)
$$

Proof: From the definition we easily have

$$
u_{\alpha}^{k} \ll u^{k}, \quad W(\alpha) \text { almost everywhere, }
$$

and

$$
M_{\alpha} \ll u, \quad W(\alpha) \text { almost everywhere. }
$$

Now from [21, p. 442]

$$
\frac{d u_{\alpha}^{k}}{d u^{k}} \underset{k \rightarrow \infty}{\rightarrow} \frac{d u_{\alpha}}{d u}, \quad u \text { and } W(\alpha) \text { almost everywhere. }
$$

\section{Hence}

$$
\frac{d u_{\alpha}^{k}(x) / d x^{k}}{d u^{k}(x) / d x^{k}} \underset{k \rightarrow \infty}{\rightarrow} \frac{d u_{\alpha}}{d u}
$$

$u$ and $W(\alpha)$ almost everywhere.

Now since $d u_{\alpha} / d u$ is a Radon-Nikodym derivative it is finite $u$ almost everywhere. Also

$$
\begin{aligned}
u_{\alpha}\left\{x \in \mathbb{R}^{\infty}: \frac{d u_{\alpha}}{d u}=0\right\} & \triangleq u_{\alpha}\left\{C_{\alpha}\right\}=\int_{C_{\alpha}} d u_{\alpha} \\
& =\int_{C_{\alpha}} \frac{d u_{\alpha}}{d u} d u=0 .
\end{aligned}
$$

Hence

$$
\left(\frac{d u_{\alpha}^{k}(x) / d x^{k}}{d u^{k}(x) / d x^{k}}\right)^{1 / k} \underset{k \rightarrow \infty}{\rightarrow} 1
$$

$u_{\alpha}$ and $W(\alpha)$ almost everywhere.

or

$$
\begin{array}{r}
\frac{1}{k} \ln \frac{d u_{\alpha}^{k}(x)}{d x^{k}}-\frac{1}{k} \ln \frac{d u^{k}(x)}{d x^{k}} \underset{k \rightarrow \infty}{\rightarrow} 0, \\
u_{\alpha} \text { and } W(\alpha) \text { almost everywhere. }
\end{array}
$$

Now from a result shown in [22]

$$
\frac{1}{k} \ln \frac{d u_{\alpha}^{k}(x)}{d x^{k}} \underset{u_{\alpha}-\operatorname{Pr}}{\rightarrow}-H_{\alpha}
$$

Hence

$$
-\frac{1}{k} \ln \frac{d u^{k}(x)}{d x^{k}} \underset{u_{\alpha}-\operatorname{Pr}}{\rightarrow} H_{\alpha}
$$

or

$$
-\frac{r}{k+r} \ln g(x) \underset{u_{\alpha}-\operatorname{Pr}}{\rightarrow} r H_{\alpha} .
$$

Hence

$$
e^{-(r / k+r)) \ln g(x)} \underset{u_{\alpha}-\text { dist }}{\rightarrow} e^{r H_{\alpha}} .
$$

Define $y$ as a random variable with the distribution

$$
P(y \leq \beta)=\int_{\Lambda} I\left\{e^{r H_{\alpha}} \leq \beta\right\} d W(\alpha)
$$

where $I\{A\}$ is the indicator function for the set $A$. Then since

$$
\begin{aligned}
u^{k}\left(x \in \mathbb{R}^{k}:\right. & \left.e^{-(r / k+r)) \ln g(x)} \leq \beta\right) \\
& =\int_{\Lambda} u_{a}^{k}\left(x \in \mathbb{R}^{k}: e^{-(r / k+r)) \ln g(x)} \leq \beta\right) d W(\alpha) .
\end{aligned}
$$

Then (because of the convergence in distribution)

$$
u_{\alpha}^{k}\left(x \in \mathbb{R}^{k}: e^{-(r / k+r)) \ln g(x)} \leq \beta\right) \underset{k \rightarrow \infty}{\rightarrow} I\left\{e^{r H_{\alpha}} \leq \beta\right\}
$$

for $\beta \neq e^{r H_{\alpha}}$. Suppose $\int_{\alpha=\alpha_{0}} d W(\alpha)>0$; then $P(y \leq \beta)$ is discontinuous at $\beta=H \alpha_{0}$. So let $\beta=e^{r H_{\alpha_{0}}}$ such that $\int_{\alpha=\alpha_{0}} d W(\alpha)=0$. Then by dominated convergence

$$
u^{k}\left(e^{-(r /(k+r)) \ln g(x)} \leq \beta\right) \underset{k \rightarrow \infty}{\rightarrow} P(y \leq \beta) .
$$

Hence we have

$$
\exp \left[-\frac{r}{k+r} \ln g(x)\right]_{u-\text { dist }}^{\rightarrow} y .
$$

Recall that

$$
\int\|x\|^{r+\epsilon} g(x) d x<M_{k}
$$

and since $g(x)$ represents a stationary measure, from the proof of Theorem 2 given in [2] we find that for some $\delta>0$

$$
\int g(x) e^{-(r(1+\delta) / k+r)\} \ln g(x)} d x<C, \quad \text { for all } k,
$$

for some $C>\infty$. Therefore $e^{-(r /(k+r)) \ln g(x)}$ is uniformly integrable in $k$ and we have the moments converging, or

$$
\int g(x) e^{-(k \wedge k+r)) \ln g(x)} d x \underset{k \rightarrow \infty}{\rightarrow} \int_{\Lambda} e^{r H_{\alpha}} d W(\alpha)=E\{y\} .
$$

Hence

$$
\|g\|_{k / k+r} \rightarrow \int_{k \rightarrow \infty} e^{+r H_{\alpha}} d W(\alpha) .
$$

Corollary:

$$
\int \frac{f_{\alpha}(x)}{g(x)^{r / k+r}} d x\left(\int g(x)^{k / k+r}\right)^{r / k} \underset{L_{1}-W(\alpha)}{\rightarrow} e^{r H_{\alpha}} .
$$

Proof: Consider

$$
\begin{aligned}
\varlimsup_{k} \int \mid \int \frac{f_{\alpha}(x)}{g(x)^{r / k+r}} d x\left(\int g(x)^{k / k+r} d x\right)^{r / k} \\
\quad-\int \frac{f_{\alpha}(x)}{f_{\alpha}(x)^{r / k+r}} d x\left(\int f_{\alpha}^{k / k+r}(x) d x\right)^{r / k} \mid d W(\alpha) \\
=\varlimsup_{k \rightarrow \infty}\left[\|g\|_{k / k+r}-\int_{\Lambda}\left\|f_{\alpha}\right\|_{k / k+r} d W(\alpha)\right]
\end{aligned}
$$

since the first term in the absolute value is always greater than or equal to the second. This is

$$
\begin{aligned}
& \leq \varlimsup_{k}\|g\|_{k / k+r}-\frac{\lim }{k} \int\left\|f_{\alpha}\right\|_{k / k+r} d W(\alpha) \\
& \leq \int_{\Lambda} e^{r H_{\alpha}} d W(\alpha)-\int \frac{\lim }{k}\left\|f_{\alpha}\right\|_{k / k+r} d W(\alpha) \\
& =\int_{\Lambda} e^{r H_{\alpha}} d W(\alpha)-\int_{\Lambda} e^{r H_{\alpha}} d W(\alpha)=0 .
\end{aligned}
$$


Remarks: Theorem 4 tells us that the optimal constant for coding a stationary source is a weighted average of the optimal constants for the ergodic decomposition of that source. This corresponds to a result by Gray and Davisson [19]. They show that the minimum average distortion for the fixed rate coding of a stationary source is the same weighted average of the distortion-rate functions of its ergodic subsources. Hence Theorem 4 provides a bridge from quantization theory to rate-distortion theory for the high-rate coding of stationary sources.

Neuhoff $e t$ al. [20] define a weighted universal sequence of codes as follows. Let $W$ be a probahility measure on $\Lambda$. Let $\rho\left(C_{k} \mid \alpha\right)$ be the distortion of a rate $R k$-dimensional source code $C_{k}$ on the ergodic source $\alpha$. Then $\left\{C_{k}\right\}_{k=1}^{\infty}$ is a weighted universal sequence of codes if

$$
\lim _{k \rightarrow \infty} \int\left|\rho\left(c_{k} \mid \alpha\right)-D_{\alpha}(R)\right| d W(\alpha)=0,
$$

where $D_{\alpha}(R)$ is the distortion rate function for the ergodic source $\alpha$. The corollary says that one quantizer matched to the mixture probability density $g(x)$ should perform well for each of the ergodic sources composing that mixture if the block length $k$ is large enough. Note for for $r=2$ $D_{\alpha}(R) \cong \exp \left[-2\left(R-H_{\alpha}\right)\right] / 2 \pi e$ for large $R$, from the Shannon lower bound to the rate distortion function, which is known to be tight for large $R$. From Theorem 3 we know that a quantizer matched to $g(x)$ operating on $\alpha$ should have large-rate performance of approximately (note $R=$ $(1 / k) \log N)$

$$
\begin{array}{r}
\rho\left(C_{k} \mid \alpha\right) \cong \exp [-2 R] J_{k, 2}\left(\int \frac{f_{\alpha}(x)}{g(x)^{2 / k+2}} d x\right) \\
\cdot\left(\int g(x)^{k / k+2}\right)^{2 / k} .
\end{array}
$$

Zador [12] shows that $\lim _{k \rightarrow \infty} J_{k, 2}=1 / 2 \pi e$. The corollary shows

$$
\left(\int \frac{f_{\alpha}(x)}{g(x)^{2 / k+2}} d x\right)\left(\int g(x)^{k / k+2}\right)^{2 / k} \underset{L_{1}-W(\alpha)}{\rightarrow} e^{2 H_{\alpha}}
$$

Hence for large rates a sequence (in $k$ ) of quantizers matched to $g$ are a weighted universal sequence.

\section{ACKNOWLEDGMENT}

I would like to thank Gary Wise of the University of Texas for many helpful discussions and especially for his assistance in the proof of Theorem 4 .

\section{APPENDIX A}

We will need the following identity which may be verified by a simple differentiation: where the right-hand side is minimized for

$$
R_{E}=\left(\int_{E} g(x)^{k / k+r} d x\right) / \int_{\mathbb{R}^{k}} g(x)^{k / k+r} d x .
$$

Take $0 / 0=0$; and $E$ is any Borel set in $\mathbb{R}^{k}$.

Proof of Lemma 2: Let $E$ be a simply connected bounded open set in $\mathbb{R}^{k}$. If $\int_{E} g(x) d x=0$ the lemma holds trivially so assume $\int_{E} g(x) d x>0$. Then from Theorem 1 we have the inequality

$$
\begin{aligned}
& \varlimsup_{N} \int_{E} N^{r / k}\left\|x-Q_{N}(x)\right\|^{r} g(x) d x \\
& \quad+\varlimsup_{N} \int_{\mathbb{R}^{k}-E} N^{r / k}\left\|x-Q_{N}(x)\right\|^{r} g(x) d x \geq J_{k, r}\|g\|_{k / k+r} .
\end{aligned}
$$

Define $N_{A}(N) \triangleq$ number of output levels of $Q_{N}(x)$ contained in A. Suppose

$$
\varlimsup_{N \rightarrow \infty} \int_{E} N^{r / k}\left\|x-Q_{N}(x)\right\|^{r} g(x) d x>M_{g}(E) .
$$

Let $\left\{N^{\prime}\right\}$ be a subsequence of $N$ such that the above $\overline{\lim }$ is

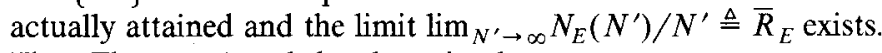
Then Theorem 1 and the above imply

$$
\lim _{N^{\prime} \rightarrow \infty} \int_{\mathbb{R}^{k}-E}\left(N^{\prime}\right)^{r / k}\left\|x-Q_{N}(x)\right\|^{r} g(x) d x<M_{g}\left(\mathbb{R}^{k}-E\right) .
$$

Let $Q_{N}^{s}(x)$ have the same output levels as $Q_{N}(x)$, plus $M_{\delta}$ more output levels distributed inside the set $E_{\delta}-E$, where

$$
E_{\delta}=\left\{X \in \mathbb{R}^{k}: \operatorname{dist}(x, E)<\delta>0\right\} ;
$$

make this such that if $x \in \mathbb{R}^{k}-\mathbb{R}^{k}-E_{\delta}$ then the closest output level from $Q_{N}^{s}(x)$ lies in $\mathbb{R}^{k}-E$. Therefore

$$
\begin{aligned}
\lim _{N^{\prime} \rightarrow \infty} \int_{\mathbb{R}^{k}-E}\left(N^{\prime}\right)^{r / k}\left\|x-Q_{N^{\prime}}(x)\right\|^{r} g(x) d x \\
\geq \varlimsup_{N^{\prime} \rightarrow \infty} \int_{\mathbb{R}^{k}-E_{\delta}}\left(N^{\prime}\right)^{r / k}\left\|x-Q_{N^{\prime}}^{s}(x)\right\|^{r} g(x) d x .
\end{aligned}
$$

Now there are at most

$$
\left(1-\frac{N_{E}\left(N^{\prime}\right)}{N^{\prime}}\right) N^{\prime}+M_{\delta} \triangleq N_{\delta}^{\prime \prime}
$$

output levels in $Q_{N}^{s},(x)$ that will have any effect on quantizing a point in $\mathbb{R}^{k}-E_{\delta}$. Therefore the right-hand side of the above inequality becomes (if $G\left(\mathbb{R}^{k}-G_{\delta}\right)=0$ set right-hand side equal to zero)

$$
\begin{gathered}
\varlimsup_{N^{\prime}, \infty} \frac{\left(N^{\prime}\right)^{r / k}}{\left(N_{\delta}^{\prime \prime}\right)^{r / k}} G\left(\mathbb{R}^{k}-E_{\delta}\right)\left(N_{\delta}^{\prime \prime}\right)^{r / k} \\
\cdot \int_{\mathbb{R}^{k}-E_{\delta}} \| x-\frac{Q_{N^{\prime}}^{s}(x) \|^{r} g(x) d x}{G\left(\mathbb{R}^{k}-E_{\delta}\right)} \\
\geq \frac{J_{k, r}\left(\int_{\mathbb{R}^{k}-E_{\delta}} g(x)^{k / k+r} d x\right)^{(k+r) / k}}{\left(1-\bar{R}_{E}\right)^{r / k}},
\end{gathered}
$$

$$
J_{k, r}\|g\|_{k / k+r}=\min _{R_{E} \in[0,1]}\left[\frac{J_{k, r}\left(\int_{E} g(x)^{k / k+r} d x\right)^{(r+k) / k}}{R_{E}^{r / k}}+J_{k, r} \frac{\left(\int_{\mathbb{R}^{k}-E} g(x)^{k / k+r} d x\right)^{(k+r) / k}}{\left(1-R_{E}\right)^{r / k}}\right],
$$


where $G(A)=\int_{A} g(x) d x$ from Theorem 1. Hence letting $\delta \rightarrow 0$ gives

$$
M_{g}\left(\mathbb{R}^{k}-E\right)>J_{k, r} \frac{\left(\int_{\mathbb{R}^{k}-E} g(x)^{k / k+r} d x\right)^{(k+r) / k}}{\left(1-\bar{R}_{E}\right)^{r / k}} .
$$

A similar construction yields

$$
\begin{aligned}
\lim _{N^{\prime} \rightarrow \infty} \int_{E}\left(N^{\prime}\right)^{r / k}\left\|x-Q_{N^{\prime}}(x)\right\|^{r} g(x) d x & \\
\geq & \frac{J_{k, r}\left(\int_{E} g(x)^{k / k+r} d x\right)^{(k+r) / k}}{\bar{R}_{E}^{r / k}} .
\end{aligned}
$$

Therefore

$J_{k, r}\|g\|_{k / k+r}$

$$
\begin{aligned}
= & \lim _{N^{\prime} \rightarrow \infty} \int_{E}\left(N^{\prime}\right)^{r / k}\left\|x-Q_{N^{\prime}}(x)\right\|^{r} g(x) d x \\
& +\lim _{N^{\prime} \rightarrow \infty} \int_{\mathbb{R}^{k}-E}\left(N^{\prime}\right)^{r / k}\left\|x-Q_{N^{\prime \prime}}(x)\right\|^{r} g(x) d x \\
> & J_{k, r}\left[\frac{\left(\int_{E} g(x)^{k / k+r} d x\right)^{(k+r) / k}}{\bar{R}_{E}^{r / k}}\right] \\
& \left.+\frac{\left(\int_{\mathbb{R}^{k}-E} g(x)^{k / k+r} d x\right)^{(k+r) / k}}{\left(1-\bar{R}_{E}\right)^{r / k}}\right]
\end{aligned}
$$

which is a contradiction by (A1). Hence

$$
\varlimsup_{N \rightarrow \infty} \int_{E} N^{r / k}\left\|x-Q_{N}(x)\right\|^{r} g(x) d x \leq M_{g}(E) .
$$

Now let $N^{\prime}$ be a subsequence of $N$ such that

$$
\begin{aligned}
\lim _{N^{\prime} \rightarrow \infty} \int_{E}\left(N^{\prime}\right)^{r / k} \| x & -Q_{N^{\prime}}(x) \|^{r} g(x) d x \\
& =\frac{\lim }{N} \int_{E}\left(N^{\prime}\right)^{r / k}\left\|x-Q_{N^{\prime}}(x)\right\|^{r} g(x) d x
\end{aligned}
$$

and such that

$$
\lim _{N^{\prime} \rightarrow \infty} N_{E}\left(N^{\prime}\right) / N^{\prime}=\boldsymbol{R}_{E} \quad \text { exists. }
$$

A similar construction to the one given above yields

$$
\begin{aligned}
& \frac{\lim }{N} \int_{E} N^{r / k}\left\|x-Q_{N}(x)\right\|^{r} g(x) d x \\
& \geq \frac{J_{k, r}\left(\int_{E} g(x)^{k / k+r} d x\right)^{(k+r) / k}}{\boldsymbol{R}_{E}^{r / k}} .
\end{aligned}
$$

Similarly

$$
\begin{aligned}
& \frac{\lim }{N} \int_{\mathbb{R}^{k}-E} N^{r / k}\left\|x-Q_{N}(x)\right\|^{r} g(x) d x \\
& \geq \frac{J_{k, r}\left(\int_{\mathbb{R}^{k}-E^{2}} g(x)^{k / k+r} d x\right)^{(k+r) / k}}{\left(1-R_{E}\right)^{r / k}} .
\end{aligned}
$$

Therefore

$$
\frac{\lim }{N} \int_{E}(\cdot)+\frac{\lim }{N} \int_{\mathbf{R}^{k}-E}(\cdot) \geq J_{k, r}\|g\|_{k / k+r}
$$

by (A1). Suppose

$$
\frac{\lim }{N} \int_{E}(\cdot)>\frac{J_{k, r}\left(\int_{E} g(x)^{k / k+r} d x\right)^{(k+r) / k}}{\boldsymbol{R}_{E}^{r / k}}
$$

which implies

$$
\begin{aligned}
& \frac{\lim }{N} \int_{\mathbf{R}^{k}}(\cdot)= J_{k, r}\|g\|_{k / k+r} \geq \underline{\lim } \int_{E}(\cdot)+\underline{\lim } \int_{\mathbb{R}^{k}-E} \\
&> \frac{J_{k, r}\left(\int_{E} g(x)^{k / k+r} d x\right)^{(k+r) / k}}{\boldsymbol{R}_{E}^{r / k}} \\
&+\frac{J_{k, r}\left(\int_{\mathbf{R}^{k}-E} g(x)^{k / k+r} d x\right)^{(k+r) / k}}{\left(1-\boldsymbol{R}_{E}\right)^{r / k}} \\
& \geq J_{k, r}\|g\|_{k / k+r}
\end{aligned}
$$

which is a contradiction. Therefore

$\underline{\lim } \int_{E} N^{r / k}\left\|x-Q_{N}(x)\right\|^{r} g(x) d x$

Suppose

$$
=\frac{J_{k, r}\left(\int_{E} g(x)^{k / k+r} d x\right)^{(k+r) / k}}{\boldsymbol{R}_{E}^{r / k}} .
$$

$$
\boldsymbol{R}_{E} \neq \int_{E} g(x)^{k / k+r} d x / \int_{\mathbf{R}^{k}} g(x)^{k / k+r} d x
$$

Then again

$$
\begin{aligned}
J_{k, r}\|g\|_{k / k+r}= & \lim _{N} \int_{\mathbf{R}^{k}} N^{r / k}\left\|x-Q_{N}(x)\right\|^{r} g(x) d x \\
\geq & \frac{\lim }{N} \int_{E}(\cdot)+\frac{\lim }{N} \int_{\mathbf{R}^{k}-E}(\cdot) \\
= & \frac{J_{k, r}\left(\int_{E} g(x)^{k / k+r} d x\right)^{(k+r) / k}}{\boldsymbol{R}_{E}^{r / k}} \\
& +\frac{J_{k, r}\left(\int_{\mathbf{R}^{k}-E^{k}} g(x)^{k / k+r} d x\right)^{(k+r) / k}}{\left(1-\boldsymbol{R}_{E}\right)^{r / k}}
\end{aligned}
$$

and if $\boldsymbol{R}_{E}$ is not optimum we have a contradiction from (A1). Therefore $\boldsymbol{R}_{E}$ is optimum and

$$
\frac{\lim }{N} N^{k / r} \int_{E}\left\|x-Q_{N}(x)\right\|^{r} g(x) d x=M_{g}(E)
$$

implies with (2) that the limit exists. Contained above is the fact that the limit also exists for the complement of $E\left(\mathbb{R}^{k}-E\right)$. Therefore

$$
\lim _{N \rightarrow \infty} M_{g}^{N}(E)=M_{g}(E)
$$

for all simply connectcd bounded open sets $E$. 
Now let $E$ be an arbitrary open set. Define

$H\left(i_{1}, i_{2}, \cdots, i_{k}\right)$

$=\left\{x: i_{1}<X_{1}<i_{1}+1, i_{2}<X_{2}<i_{2}+1, \cdots, i_{k}<X_{k}<i_{k}+1\right\}$

$=H(i)$

where $\boldsymbol{i}=\left(i_{1}, i_{2}, \cdots, i_{k}\right)$ and $\mathbb{R}^{k}=\left(\bigcup_{i} H(\boldsymbol{i})\right) \cup B$, where $B$ is a boundary set of Borel measure zero. Define $E_{i}=H(i) \cap E$. Hence $E=\left(\cup_{i} E_{i}\right) \cup B_{E}$ and $B_{E}$ has measure zero. Each $E_{i}$ is a bounded open set. Therefore it may be represented as a countable union of disjoint bounded simply connected sets. Since there are only a countable number of $E_{i}$ we may represent $E$ as

$$
E=\left(\bigcup_{i=1}^{\infty} E_{i}^{\prime}\right) \cup B_{E},
$$

where the $E_{i}^{\prime}$ are simply connected bounded open sets. Now since

$$
\begin{aligned}
\frac{\lim }{N} M_{g}^{N}(E) & =\frac{\lim }{N} \sum_{i=1}^{\infty} M_{g}^{N}\left(E_{i}\right) \geq \sum_{i=1}^{\infty} \frac{\lim }{N} M_{g}^{N}\left(E_{i}\right) \\
& =\sum_{i=1}^{\infty} M_{g}^{N}\left(E_{i}\right)=M(E)
\end{aligned}
$$

then

$$
\frac{\lim }{N} M_{g}^{N}(E) \geq M(E)
$$

for all open sets $E$.

Suppose for some subsequence $N^{\prime}$ the following limit exists and satisfies $\lim _{N^{\prime}} M_{g}^{N^{\prime}}(E)>M_{g}(E)$ but that

$$
\lim _{N^{\prime}} M_{g}^{N^{\prime}}\left(\mathbb{R}^{k}-E\right)+\lim _{N^{\prime}} M_{g}^{N^{\prime}}(E)=M_{g}\left(\mathbb{R}^{k}\right)
$$

which implies $\lim _{N^{\prime}} M_{g}^{N^{\prime}}\left(\mathbb{R}^{k}-E\right)<M_{g}\left(\mathbb{R}^{k}-E\right)$. Let $\partial E$ denote the boundary of $E$ and $\bar{E} \triangleq \partial E \cup E$. Now since $M_{g}^{N^{\prime}}(\partial E)$ $=M_{g}^{N}(\partial E)=0$ for all $N$ we have

$$
\lim _{N^{\prime}} M_{g}^{N^{\prime}}\left(\mathbb{R}^{k}-\bar{E}\right)=\lim _{N^{\prime}} M_{g}^{N^{\prime}}\left(\mathbb{R}^{k}-E\right)<M_{g}\left(\mathbb{R}^{k}-\bar{E}\right) .
$$

But $\mathbb{R}^{k}-\bar{E}$ is open, hence

$$
\lim _{N^{\prime}} M_{g}^{N^{\prime}}\left(\mathbb{R}^{k}-\bar{E}\right) \geq M_{g}\left(\mathbb{R}^{k}-\bar{E}\right),
$$

which is a contradiction. Hence $\lim _{N \rightarrow \infty} M_{g}^{N}(E)=M_{g}(E)$ for all open $E$. Suppose $E$ is a Borel set. Then there exists a sequence of open sets $E_{i}$ such that $E \subset E_{i}$ for all $i$ and $M_{g}\left(E_{i}\right)=$ $\lim _{i \rightarrow \infty} M_{g}(E)$. Then $\varlimsup_{\lim _{N}} M_{g}^{N}(E) \leq \varlimsup_{\lim _{N}} M_{g}^{N}\left(E_{i}\right)=M_{g}\left(E_{i}\right)$ for all $i$ implies $\varlimsup_{N} M_{g}^{N}(E) \leq M_{g}(E)$. Suppose $\overline{\lim }_{N} M_{g}^{N}(E)<$ $M_{g}(E)$. Then since

$$
\begin{aligned}
\varlimsup_{N} M_{g}^{N}(E)+\varlimsup_{N} M_{g}^{N} & \left(\mathbb{R}^{k}-E\right) \\
& \geq M_{g}\left(\mathbb{R}^{k}\right)=M_{g}(E)+M_{g}\left(\mathbb{R}^{k}-E\right),
\end{aligned}
$$

this would imply $\varlimsup_{N} M_{g}^{N}\left(\mathbb{R}^{k}-E\right)>M_{g}\left(\mathbb{R}^{k}-E\right)$. But $\mathbb{R}^{k}-E$ is Borel, hence from above we know $\varlimsup_{N} M_{g}^{N}\left(\mathbb{R}^{k}-E\right) \leq M_{g}\left(\mathbb{R}^{k}\right.$ $-E$ ), which is a contradiction.

Suppose $E$ is a Lebesgue measurable set. Then we may write $E=E^{\prime} \cup Z$, where $E^{\prime}$ is a Borel measurable set and is disjoint from $Z$ which is a measurable set of Lebesgue measure zero. Obviously for every $N, M_{g}^{N}(Z)=0=M_{g}(Z)$. Hence

$$
\lim _{N \rightarrow \infty} M_{g}^{N}(E)=\lim _{N \rightarrow \infty} M_{g}^{N}\left(E^{\prime}\right)=M_{g}\left(E^{\prime}\right)=M_{g}(E) .
$$

Hence the lemma is proved.

\section{APPENDIX B}

We define an $N$ level quantizer on the interval $[0,1]$ as follows. Place $N-1$ output levels uniformly on the subinterval $\left[y_{N}, 1\right]$ and one output level at $y_{N}$. If a sample point is in $\left[0, y_{N}\right)$ the output is $y_{N}$. If a sample point is in $\left[y_{N}, 1\right]$ use the $N-1$ level uniform quantizer to quantize it. Note this is not a nearest neighbor quantizer. Now choose a $\delta$ and an $\varepsilon$ such that $1>\delta>0$, $1 / 3>\epsilon>0$ and $2 \epsilon+\frac{2}{3} \delta+\epsilon \delta<2 / 3$.

Let $y_{N}=1 / N^{(2 / 3)+\epsilon}$. Suppose $g(x)=1$ on $[0,1]$. Then

$$
\begin{aligned}
N^{2} \int_{0}^{1} \mid x- & \left.Q_{N}(x)\right|^{2} d x \\
& =N^{2} \frac{\left(1-y_{N}\right)^{3}}{(N-1)^{2} 12}+N^{2} \int_{0}^{y_{N}}\left(y_{N}-x\right)^{2} d x \\
& =\frac{N^{2}\left(1-y_{N}\right)^{3}}{12(N-1)^{2}}+\frac{y_{N}^{3} N^{2}}{3} \underset{N \rightarrow \infty}{\rightarrow} \frac{1}{12}=J_{1,2}\|y\|_{1 / 3} .
\end{aligned}
$$

So this quantizer gives the optimum asymptotic constant.

Now let $f(x)=\delta / x^{1-\delta}$.

$$
\begin{aligned}
& N^{2} \int_{0}^{1}\left|x-Q_{N}(x)\right|^{2} f(x) \\
& \geq N^{2} \delta \int_{0}^{y_{N}}\left(y_{N}-x\right)^{2} / x^{1-\delta} d x \\
& =\delta N^{2} C_{\delta} y_{N}^{2+\delta}, \quad \text { where } C_{\delta}=\left(\frac{1}{\delta}-\frac{2}{1-\delta}+\frac{1}{2+\delta}\right)>0, \\
& =\frac{\delta N^{2} C_{\delta}}{N^{(4 / 3)+2 \varepsilon+(2 / 3) \delta+\epsilon \delta}}
\end{aligned}
$$

but $2 \epsilon+(2 / 3) \delta+\epsilon \delta<(2 / 3)$ implies

$$
\frac{\lim }{N} \int_{0}^{1} N^{2}\left|x-Q_{N}(x)\right|^{2} f(x) d x=\infty
$$

even though Bennett's integral

$$
\left(\int_{0}^{1} \frac{f(x)}{g(x)^{r / k+r}} d x\right)\left(\int_{0}^{1} g(x)^{k / k+r} d x\right)^{r / k}=1 .
$$

\section{REFERENCES}

[1] P. F. Panter and W. Dite, "Quantization in pulse-count modulation with nonuniform spacing of levels," Proc. IRE, vol. 39, pp. 44-48, 1951.

[2] S. P. Lloyd, "Least squares quantization in PCM," IEEE Trans. Inform. Theory, vol. IT-28, pp. 129-136, Mar. 1982.

[3] J. A. Bucklew and G. L. Wise, "A note on multidimensional asymptotic quantization theory," in Proc. Eighteenth Ann. Allerton Conf. Commun. Contr. Comput., Oct. 8-10, 1980.

[4] B. Smith, "Instantaneous companding of quantized signals," Bell Syst. Tech. J., vol, 36, pp. 653-709, May 1957.

[5] V. R. Algazi, "Useful approximations to optimum quantization," IEEE Trans. Commun. Tech., vol. COM-14, pp. 297-301, 1966.

[6] J. Max, "Quantizing for minimum distortion," IRE Trans. Inform. Theory, vol. IT-6, pp. 7-12, Mar. 1960.

[7] R. M. Gray and A. H. Gray, Jr., "Asymptotically optimal quantizers," IEEE Trans. Inform. Theory, vol. IT 23, pp. 143 144, Jan. 1977.

[8] A. Gersho, "Asymptotically optimal block quantization," IEEE Trans. Inform. Theory, vol. IT-25, pp. 373-380, July 1979.

[9] Y. Yamada, S. Tazaki, and R. M. Gray, "Asymptotic performance of block quantizers with difference distortion measures," IEEE Trans. Inform. Theory, vol. IT-26, pp. 6-14, Jan. 1980. 
[10] H. Gish and J. N. Pierce, "Asymptotically efficient quantizing," IEEE Trans. Inform. Theory, vol. IT-14, pp. 676-683, Sept. 1968.

[11] W. R. Bennett, "Spectra of quantized signals," Bell Syst. Tech. J., vol. 27 , pp. 446-472, July, 1948.

[12] P. Zador, "Development and evaluation of procedures for quantizing multivariate distributions," Ph.D. dissertation, Stanford Univ., Stanford, CA, 1964.

[13] - "Asymptotic quantization of continuous random variables," unpublished memorandum, Bell Laboratories, 1966.

[14] P. Billingsley, Convergence of Probability Measures. New York: Wiley, 1968.

[15] N. Dunford and J. T. Schwartz, Linear Operators-Part I. New York: Wiley, 1958.

[16] H. L. Royden, Real Analysis. New York: MacMillan, 1968.

[17] V. A. Rohlin, "Lectures on the entropy theory of measure preserv- ing transformations," Russian Math. Surveys., vol. 22, no. 5, pp. $1-52,1967$.

[18] R. M. Gray and L. D. Davisson, "The ergodic decomposition of discrete stationary sources," IEEE Trans. Inform. Theory, vol. IT-20, pp. 625-636, Sept. 1974.

[19] - "Source coding theorems without the ergodic assumption," IEEE Trans. Inform. Theory, vol. IT-20, pp. 502-516, July 1974.

[20] D. L. Neuhoff, R. M. Gray, and L. D. Davisson "Fixed rate universal block source coding with a fidelity criterion," IEEE Trans. Inform. Theory, vol. IT-21, pp. 511-523, 1975.

[21] I. I. Gihman and A. V. Skorohod, The Theory of Stochastic Processes I. New York: Springer-Verlag, 1974.

[22] A. Pérez, "On the theory of information in the case of an abstract alphabet," in Trans. First Prague Conf. Inform. Theory, Stat. Dec. Fns., Rand. Processes, 1957.

\title{
Fixed-Rate Encoding of Individual Sequences with Side Information
}

\author{
JACOB ZIV, FELLOW, IEEE
}

\begin{abstract}
For every infinite sequence $x$ and a given side-information sequence $y$, we define a quality $H(x \mid y)$ called the finite-state conditional complexity of $x$ given $y$. It is shown that $H(x \mid y)$ is the smallest asymptotically attainable fixed-rate at which $x$ can be transmitted with negligibly small distortion, given $y$. Moreover, it is demonstrated that in order to achieve an arbitrary small distortion for all sequences such that $H(x \mid y)$ is less than the allowable transmission rate it is not necessary for the encoder to have access to the side-information sequence $y$ (provided it is available to the decoder). This result is a generalization of the classical Slepian-Wolf result for cases where the probabilistic characterization of $x$ and $y$ is not known, or does not exist.
\end{abstract}

\section{INTRODUCtion AND Statement OF RESUlts}

I $\mathrm{N}$ [1] we discussed the class of finite-state encoders which map, at a fixed rate, input strings drawn from a source of $\alpha$ letters into equally long strings over an alphabet of $\beta \leqslant \alpha$ letters. The aim was to minimize the number of bits per symbol $\log _{2} \beta$ while securing zero or negligibly small distortion.

In this paper the results of [1] are extended to include the case of finite-state fixed-rate encoding with side information. Our problem concerns the system shown in Fig. 1.

When an infinite input-sequence $x=x_{1}, x_{2}, x_{i}, \cdots\left(x_{i}\right.$ $\in A$ where $|A|=\alpha)$ and the side-information sequence $y=y_{1}, y_{2}, \cdots, y_{i}, \cdots\left(y_{i} \in G,|G|=\gamma\right)$ are fed into a

Manuscript received October 28, 1982; revised February 17, 1983. This work was partly done while on a sabbatical leave at Bell Telephone Laboratories, Murray Hill, NJ 07974.

The author is with the Department of Electrical Engineering, Technion - Israel Institute of Technology, Haifa, Israel.

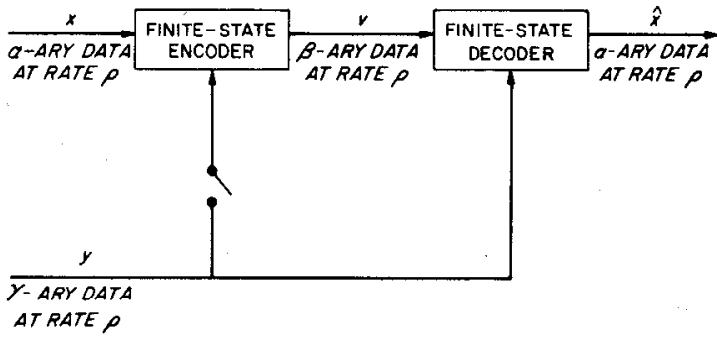

Fig. 1. A communication system with side information.

fixed-rate finite-state encoder $E=(S, A, B, G, g(\cdot), f(\cdot)$, $t)$. The encoder emits an infinite sequence $v=$ $v_{1}, v_{2}, \cdots, v_{i}, \cdots\left(v_{i} \in B,|B|=\beta\right)$ while going through an infinite sequence of states $z=z_{1}, z_{2}, \cdots, z_{i}, \cdots ; z_{i} \in S$ according to

$$
\begin{aligned}
v_{i} & =f\left(z_{i+t}, x_{i+t}, y_{i+t}\right), \quad i=1,2, \cdots, \\
z_{i+1} & =g\left(z_{i}, x_{i}, y_{i}\right),
\end{aligned}
$$

where $t$, the encoding delay, is some nonnegative integer, $z_{i}$ is the state of the encoder when it is being fed by the input symbols $x_{i}$ and $y_{i}$, and $z_{1}$ is the initial state and is a prescribed fixed member of the finite set of states $S$.

In a similar way, the decoder $D$ is a mapping of the infinite sequence $v$ into the sequence $\hat{x}=\hat{x}_{1}, \hat{x}_{2}, \cdots, \hat{x}_{i}, \cdots$ $\left(\hat{x}_{i} \in A\right)$ where

$$
\begin{aligned}
\hat{x}_{i} & =f^{\prime}\left(v_{i-t}, y_{i-t}, z_{i-t}^{\prime}\right) \\
z_{i+1}^{\prime} & =g^{\prime}\left(v_{i}, y_{i}, z_{i}^{\prime}\right)
\end{aligned}
$$

